\title{
Probing Bino-Wino coannihilation at the LHC
}

\author{
Natsumi Nagata, ${ }^{a, b}$ Hidetoshi Otono $^{c}$ and Satoshi Shirai ${ }^{d}$ \\ ${ }^{a}$ William I. Fine Theoretical Physics Institute, School of Physics and Astronomy, \\ University of Minnesota, \\ 116 Church Street S.E., Minneapolis, MN 55455, U.S.A. \\ ${ }^{b}$ Kavli Institute for the Physics and Mathematics of the Universe (WPI), \\ The University of Tokyo Institutes for Advanced Study, The University of Tokyo, \\ 5-1-5 Kashiwanoha, Kashiwa, Chiba 27\%-8583, Japan \\ ${ }^{c}$ Research Center for Advanced Particle Physics, Kyushu University, \\ 744, Motooka, Nishi-ku, Fukuoka, 819-0395, Japan \\ ${ }^{d}$ Deutsches Elektronen-Synchrotron (DESY), \\ Notkestrasse 85, D-22607 Hamburg, Germany \\ E-mail: nagat006@umn.edu, otono@phys.kyushu-u.ac.jp, \\ satoshi.shirai@desy.de
}

AbStRaCt: We study bino-wino coannihilation scenario in the so-called spread or minisplit supersymmetry. We show that, in this model, a neutral wino has a macroscopic decay length in a wide range of parameter space. This characteristic feature could be observed as a displaced vertex plus missing transverse energy event at the LHC. In this paper, we study the current constraints and future prospects on the scenario based on the displaced vertex search performed by the ATLAS collaboration. It is found that a sizable parameter region can be probed at the $8 \mathrm{TeV}$ LHC run. This search strategy will considerably extend its reach at the next stage of the LHC running, and thus play a crucial role to examine a possibility of bino dark matter in the mini-split type supersymmetric models.

KEYWORDS: Supersymmetry Phenomenology

ArXiv ePrint: 1506.08206 


\section{Contents}

1 Introduction 1

2 Wino decay 3

2.1 Effective theory for wino decay 4

2.2 Decay length in bino-wino coannihilation 9

3 LHC search $\quad 12$

$\begin{array}{lll}3.1 & \text { Previous LHC study } & 12\end{array}$

$\begin{array}{ll}3.2 \text { Signal simulation setup } & 13\end{array}$

$\begin{array}{lll}3.3 & \text { LHC prospects } & 15\end{array}$

$\begin{array}{lll}4 & \text { Conclusion and discussion } & 17\end{array}$

\section{Introduction}

Split supersymmetry (SUSY) [1-6] is an interesting candidate for physics beyond the Standard Model (SM). This class of SUSY models have a mass spectrum in which scalar particles except the SM-like Higgs boson have masses much larger than the electroweak (EW) scale, while gauginos, probably Higgsino as well, lie not far from the EW scale. With such heavy scalars, the split SUSY can overcome disadvantages of the weak-scale SUSY, such as SUSY flavor/CP problems [7] and too rapid proton decay in the minimal SUSY grand unified theory (GUT) $[8,9]$. The light fermionic SUSY partners enable the model to take over advantages of the weak-scale SUSY: the lightest SUSY particle (LSP) as a dark matter $(\mathrm{DM})$ candidate and gauge coupling unification. Thus, although the origin of the EW scale is not easily elucidated in this model, its phenomenological aspects are quite appealing.

After the discovery of the SM-like Higgs boson [10,11] with a mass of around $125 \mathrm{GeV}$ [12], this framework becomes increasingly convincing. Especially, a spectrum with mild splitting [13-19], where the scalar mass scale falls into from several tens of $\mathrm{TeV}$ to a $\mathrm{PeV}$ and the gaugino mass scale is $\mathcal{O}(1) \mathrm{TeV}$, is getting more popular - this mass spectrum is often called spread or mini-split SUSY, as the $125 \mathrm{GeV}$ Higgs mass is a sweet spot of this spectrum [20-24]. Such models can also improve gauge coupling unification [25], and accommodate a simple GUT without suffering from the rapid proton decay problem [26-28]. Moreover, reasonable assumptions on the multiverse lead to this spectrum [29].

The realization of such a mass spectrum is rather easy. We may simply assume some charge on the SUSY breaking field $X$. Then, the symmetry associated with the charge forbids Lagrangian terms $\left[X W^{\alpha} W_{\alpha} / M_{*}\right]_{\theta^{2}}$, where $W^{\alpha}$ is a gauge field strength superfield and $M_{*}$ is a cut-off scale, e.g., the Planck scale. Since these terms reduce to the gaugino 
mass terms after the SUSY breaking, absence of these terms implies suppression of gaugino masses. On the other hand, terms like $\left[X X^{\dagger} \Phi \Phi^{\dagger} / M_{*}^{2}\right]_{\theta^{4}}$, where $\Phi$ is a matter chiral superfield, are generally allowed and lead to soft scalar masses of $\widetilde{m}=\mathcal{O}\left(F_{X} / M_{*}\right)$, with $F_{X}$ the $F$-term of the SUSY breaking field $X$. The Higgsino mass $\mu$ is usually expected to be of the same order of sfermion masses: $|\mu| \sim \widetilde{m}$, though there are several models that predict a smaller value for $|\mu|$. Gaugino masses may come from the anomaly mediation mechanism [30,31] as well as threshold effects of the Higgs fields [32] or extra matter fields [33-39]. In these cases, the gaugino masses are suppressed by a loop factor compared to the scalar mass scale $\widetilde{m}$, and thus the spread/mini-split spectrum can be realized. In addition, this hierarchical mass spectrum makes the mixing among gauginos and Higgsino negligible, and thus they can be regarded as almost pure states.

If the contribution from the anomaly mediation is dominant, the LSP is a pure wino. This wino LSP has various interesting features as a DM candidate. Since winos are charged under the EW interactions, their self-annihilation cross sections are rather large, which allows a wino with a mass up to $3 \mathrm{TeV}$ to be consistent with the current observed $\mathrm{DM}$ density [40]. Such a large annihilation cross section also makes the indirect detection of wino DM through cosmic ray signals quite promising [41-46]. Direct detection of wino DM has also been intensively studied [47-51]. In addition, a pure wino offers a unique collider signal. In the spread/mini-split SUSY scenario, the mass splitting between the neutral and charged winos is predicted to be fairly small. This renders the charged wino live long, with a decay length of $c \tau_{\widetilde{W}^{0}}=\mathcal{O}(1) \mathrm{cm}$. At collider, a produced charged wino leaves a charged track of this length, which is very useful for the discovery and measurement of the wino LSP [52-54].

On the other hand, when other contributions like threshold effects are comparable to that of the anomaly mediation, it is questionable whether the wino LSP is the case or not. Actually, it turns out that an $M_{*}$ which is slightly smaller than the reduced Planck scale easily leads to the bino LSP $[14,29]$. The presence of extra matters may also favor the bino LSP, since a wino tends to receive larger quantum corrections from the extra matters due to its larger gauge coupling compared to a bino. The bino LSP is, however, often disfavored on the basis of cosmology; on the assumption of the $R$-parity conservation and conventional cosmological history, the bino LSP case usually suffers from the overproduction of DM because of its small self-annihilation cross section. For the bino abundance to be consistent with the current observation, we need to rely on some exceptional situation [55]: gaugino coannihilation or Higgs funnel. If the Higgsino mass is larger than $\mathcal{O}(10) \mathrm{TeV}$, the Higgs funnel cannot work effectively since the bino-Higgs coupling is highly suppressed. In this case, gaugino coannihilation is the only possibility. We previously studied the coannihilation of the bino LSP with a gluino [56]. In this work, we focus on the bino-wino coannihilation [57-60].

The bino DM is quite sterile compared to the wino DM, since both the self-annihilation cross section and the direct detection rate are suppressed by heavy masses of sfermions and Higgsino. Thus, probing this spectrum with the DM experiments is extremely challenging. Instead, the bino-wino coannihilation scenario has a specific mass spectrum; wino should have a mass fairly close to the bino mass in order to make coannihilation effec- 
tive and to assure that the relic abundance of the bino DM is less than or equal to the observed DM density. Previous works have revealed that the mass difference $\Delta M$ should be $\lesssim \mathcal{O}(10) \mathrm{GeV}$ [57-60] to satisfy the condition. Such a small mass difference makes it possible to probe the scenario in collider experiments, since a degenerate mass spectrum often gives rise to a long-lived particle, which offers a distinct signature. Indeed, we find that a neutral wino can actually be long-lived in our setup and thus be a nice target to probe the scenario.

In this work, we study in detail the decay of a neutral wino in the bino-wino coannihilation scenario and show that it typically has a decay length larger than $\mathcal{O}(1) \mathrm{mm}$. Then, we consider the detectability of the neutral wino decay at the LHC. It is found that searches for a displaced vertex (DV) can actually be a powerful probe for a neutral wino with such a long decay length, and thus provide a promising way of testing the bino-wino coannihilation scenario.

This paper is organized as follows. In the next section, we formulate an effective theory for bino and wino to study the wino decay in the spread/mini-split spectrum. Then, in section 3, we discuss the current constraints and future prospects on the searches for the decay signature of a long-lived neutral wino. Finally, section 4 is devoted to conclusion and discussion.

\section{Wino decay}

In this section, we discuss the decay properties of a neutral wino in the case where the neutral wino is highly degenerate with the bino LSP in mass and Higgsino and sfermions are much heavier than these EW gauginos. To adequately deal with this hierarchical setup, in section 2.1, we first construct a low-energy effective theory for the EW gauginos by integrating out these heavy particles. Then, in section 2.2 , we discuss the decay properties of the neutral wino. We also show expected values for its decay length taking into account the thermal relic abundance of the bino LSP.

Before going into the detailed discussion, let us first summarize the results of this section. In figure 1, we show the mass spectrum for the EW gauginos and the suppression factors in their decay rates. We assume the bino-wino mass difference, $\Delta M$, to be $\mathcal{O}(10) \mathrm{GeV}$, which leads to successful DM coannihilation [57-60]. The decay rate of wino into the bino LSP is suppressed by the heavy Higgsino mass. Charged wino can decay into bino promptly for $|\mu|<\mathcal{O}(10) \mathrm{PeV}$, since the decay occurs via a dimension-five operator. On the other hand, the neutral wino decay is not so rapid. If $\Delta M$ is less than the $Z$ boson mass, neutral wino decays into bino only through the virtual $Z$ boson or Higgs boson $h$ exchange, or via the two-body decay process with emitting a photon at loop level. As we see below, the $Z$ boson mediated decay and the two-body photon-emitting processes are suppressed by a factor of $|\mu|^{-4}$. Regarding the Higgs boson mediated decay, on the other hand, its decay rate is only suppressed by a factor of $|\mu|^{-2}$, though the small couplings between the Higgs boson and the SM fermions prevent neutral wino from decaying rapidly. As a result, for $|\mu| \gtrsim 10 \mathrm{TeV}$, the decay length $c \tau_{\widetilde{W}^{0}}$ of neutral wino gets macroscopic: $c \tau_{\widetilde{W}^{0}} \gtrsim \mathcal{O}(1) \mathrm{mm}$. 


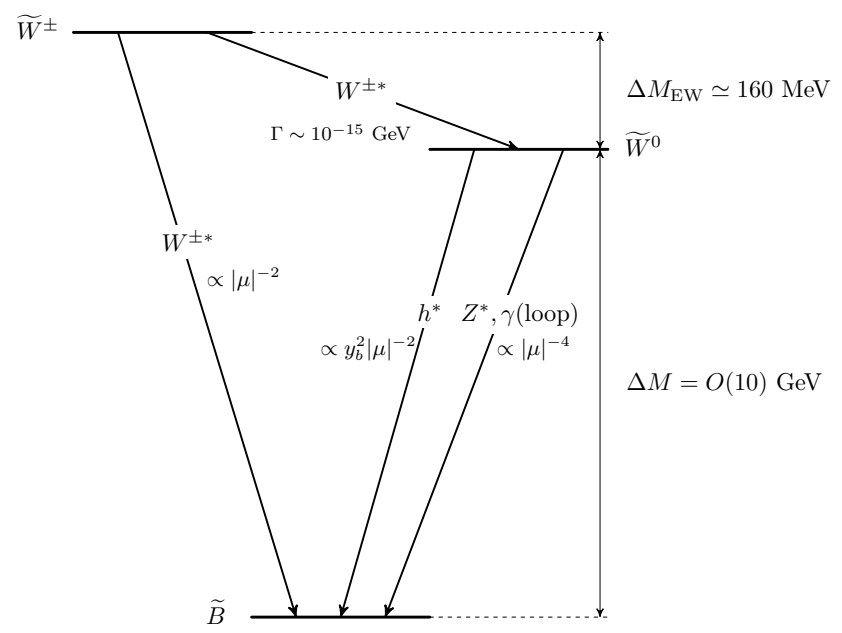

Figure 1. Mass spectrum and decay chains of the present model.

\subsection{Effective theory for wino decay}

Here, we discuss the wino decay based on the effective field theoretical approach. To begin with, we introduce the full theory containing Higgsino with renormalizable interactions. Then, we obtain a relevant effective field theory by integrating out the heavy Higgsino. The Higgsino contributions are described by higher-dimensional operators in the effective theory, which causes the wino decay into the bino LSP.

Full theory above Higgsino scale. First, let us consider the full theory. In the minispit/spread SUSY, it is reasonable to assume that the sfermion mass scale $\widetilde{m}$ is similar to or greater than the Higgsino mass $\mu: \widetilde{m} \gtrsim|\mu|$. In this case, the decay of wino is dominantly controlled by the gaugino-Higgsino-Higgs couplings, rather than the interactions with sfermions and heavy Higgs bosons, and thus we can safely neglect their contributions in the following discussion.

The gaugino-Higgsino-Higgs interactions are given by

$$
\begin{aligned}
\mathcal{L}_{\text {int }}= & -\frac{1}{\sqrt{2}}\left\{g_{1 u} H^{\dagger} \widetilde{H}_{u}+g_{1 d} \epsilon^{\alpha \beta}(H)_{\alpha}\left(\widetilde{H}_{d}\right)_{\beta}\right\} \widetilde{B} \\
& -\sqrt{2}\left\{g_{2 u} H^{\dagger} T^{A} \tilde{H}_{u}-g_{2 d} \epsilon^{\alpha \beta}(H)_{\alpha}\left(T^{A} \widetilde{H}_{d}\right)_{\beta}\right\} \widetilde{W}^{A}+\text { h.c. },
\end{aligned}
$$

where $\widetilde{H}_{u, d}, \widetilde{B}$, and $\widetilde{W}^{A}(A=1,2,3)$ denote the Higgsino, bino, and wino fields, respectively; $H$ is the SM Higgs field; $T^{A}$ are the $\mathrm{SU}(2)_{L}$ generators. In this paper, we mainly use the two-component notation for fermion fields unless otherwise noted. At the leading order, the above coupling constants are given by

$$
\begin{array}{ll}
g_{1 u}=g^{\prime} \sin \beta, & g_{1 d}=g^{\prime} \cos \beta, \\
g_{2 u}=g \sin \beta, & g_{2 d}=g \cos \beta,
\end{array}
$$

at the SUSY breaking scale $\widetilde{m}$. Here, $g^{\prime}$ and $g$ are the $\mathrm{U}(1)_{Y}$ and $\mathrm{SU}(2)_{L}$ gauge coupling constants, respectively, and $\tan \beta \equiv\left\langle H_{u}^{0}\right\rangle /\left\langle H_{d}^{0}\right\rangle$. 
The gaugino and Higgsino mass terms are defined by

$$
\mathcal{L}_{\text {mass }}=-\frac{M_{1}}{2} \widetilde{B} \widetilde{B}-\frac{M_{2}}{2} \widetilde{W}^{A} \widetilde{W}^{A}-\mu \epsilon^{\alpha \beta}\left(\widetilde{H}_{u}\right)_{\alpha}\left(\widetilde{H}_{d}\right)_{\beta}+\text { h.c. },
$$

with $\epsilon^{\alpha \beta}$ the antisymmetric tensor. In the following subsection, we construct an effective filed theory for the gauginos by integrating out the Higgsinos $\widetilde{H}_{u}$ and $\widetilde{H}_{d}$, which are supposed to be much heavier than the gauginos.

Effective theory below Higgsino scale. Next, we formulate an effective theory which describes the wino decay into the bino LSP. The decay is caused by effective interactions expressed by higher-dimensional operators, which are induced when we integrate out heavier particles than gauginos - Higgsinos and scalar particles whose masses are $\mathcal{O}\left(10-10^{3}\right) \mathrm{TeV}$. Let us write down relevant operators up to dimension six. For the dimension-five operators, we have

$$
\begin{aligned}
\mathcal{O}_{1}^{(5)} & =\widetilde{B} \widetilde{W}^{A} H^{\dagger} T^{A} H, \\
\mathcal{O}_{2}^{(5)} & =\widetilde{B} \sigma^{\mu \nu} \widetilde{W}^{A} W_{\mu \nu}^{A}, \\
\mathcal{Q}_{1} & =\frac{1}{2} \widetilde{B} \widetilde{B}|H|^{2}, \\
\mathcal{Q}_{2} & =\frac{1}{2} \widetilde{W^{A}} \widetilde{W}^{A}|H|^{2},
\end{aligned}
$$

where $W_{\mu \nu}^{A}$ is the $\mathrm{SU}(2)_{L}$ gauge field strength tensor; $\sigma_{\mu \nu} \equiv \frac{i}{2}\left(\sigma_{\mu} \bar{\sigma}_{\nu}-\sigma_{\nu} \bar{\sigma}_{\mu}\right)$, where $\sigma^{\mu}=$ $\left(\sigma^{0}, \sigma^{i}\right)$ and $\bar{\sigma}^{\mu}=\left(\sigma^{0},-\sigma^{i}\right)$ with $\sigma^{i}(i=1,2,3)$ the Pauli matrices. The first two operators contain a bino and a wino, and thus directly contribute to the wino decay into the bino LSP. The latter two are, on the other hand, only relevant to the mass matrix for the neutral bino and wino; these operators reduce to the mass terms for them after the EW symmetry breaking. As for dimension-six, we have

$$
\mathcal{O}^{(6)}=\widetilde{B}^{\dagger} \bar{\sigma}^{\mu} \widetilde{W}^{A} H^{\dagger} T^{A} i \overleftrightarrow{D_{\mu}}{ }_{\mu}
$$

where $D_{\mu}$ is the covariant derivative and $A \overleftrightarrow{D}_{\mu} B \equiv A D_{\mu} B-\left(D_{\mu} B\right) A$ with $A$ and $B$ arbitrary fields. This operator also contributes to the wino decay, though its effect is further suppressed by a heavy mass scale. Then, the effective interactions are given as follows:

$$
\Delta \mathcal{L}_{\text {int }}=\sum_{i=1,2} C_{i}^{(5)} \mathcal{O}_{i}^{(5)}+\sum_{i=1,2} \widetilde{C}_{i} \mathcal{Q}_{i}+C^{(6)} \mathcal{O}^{(6)}+\text { h.c. }
$$

The Wilson coefficients of these operators are determined below.

By evaluating the tree-level Higgsino exchange diagrams (figure 2a), we readily obtain

$$
\begin{aligned}
C_{1}^{(5)} & =\frac{1}{\mu}\left(g_{1 u} g_{2 d}+g_{1 d} g_{2 u}\right)+\frac{1}{2|\mu|^{2}}\left[\left(g_{1 u}^{*} g_{2 u}+g_{1 d}^{*} g_{2 d}\right) M_{1}+\left(g_{1 u} g_{2 u}^{*}+g_{1 d} g_{2 d}^{*}\right) M_{2}\right], \\
C^{(6)} & =-\frac{1}{2|\mu|^{2}}\left(g_{1 u}^{*} g_{2 u}-g_{1 d}^{*} g_{2 d}\right), \\
\widetilde{C}_{1} & =\frac{g_{1 u} g_{1 d}}{\mu}+\frac{M_{1}}{2|\mu|^{2}}\left(\left|g_{1 u}\right|^{2}+\left|g_{1 d}\right|^{2}\right), \\
\widetilde{C}_{2} & =\frac{g_{2 u} g_{2 d}}{\mu}+\frac{M_{2}}{2|\mu|^{2}}\left(\left|g_{2 u}\right|^{2}+\left|g_{2 d}\right|^{2}\right) .
\end{aligned}
$$




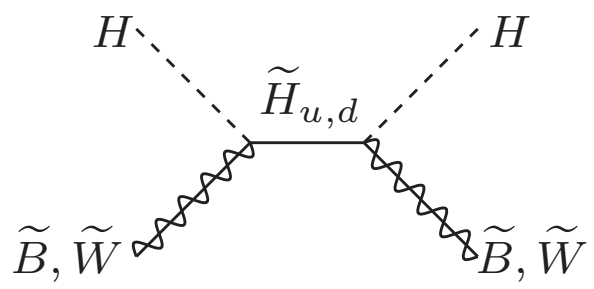

(a) Tree level

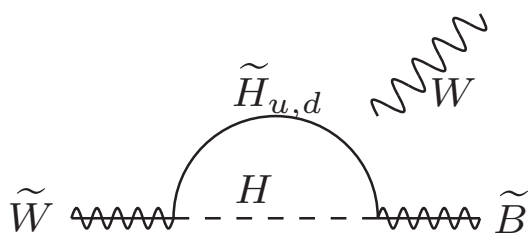

(b) One loop level

Figure 2. Examples of the diagrams, which generate the effective operators.

Here we have kept effective operators up to dimension six, and used equations of motions for external gaugino fields to eliminate redundant operators. The operator $\mathcal{O}_{2}^{(5)}$ is not induced at tree level. However, since this operator gives rise to the two-body decay process $\widetilde{W}^{0} \rightarrow \widetilde{B}+\gamma$, it could be important even though it is induced at loop level [61-64], especially when the wino mass is close to the bino mass. Thus, only for this operator, we also consider the one-loop contribution. The one-loop Higgsino-Higgs loop diagram (figure $2 \mathrm{~b}$ ) yields

$$
\begin{aligned}
C_{2}^{(5)}= & +\frac{g}{2(4 \pi)^{2} \mu}\left(g_{1 u} g_{2 d}-g_{1 d} g_{2 u}\right) \\
& -\frac{g}{8(4 \pi)^{2}}\left[\left(g_{1 u}^{*} g_{2 u}-g_{1 d}^{*} g_{2 d}\right) \frac{M_{1}}{|\mu|^{2}}-\left(g_{1 u} g_{2 u}^{*}-g_{1 d} g_{2 d}^{*}\right) \frac{M_{2}}{|\mu|^{2}}\right],
\end{aligned}
$$

where again we have kept terms up to $\mathcal{O}\left(|\mu|^{-2}\right)$. Note that the first term vanishes if we use the tree-level relation eq. (2.2). We also find that the heavy Higgs contribution of $\mathcal{O}\left(\mu^{-1}\right)$ vanishes in a similar manner. Thus, although the operator $\mathcal{O}_{2}^{(5)}$ is dimension five, its Wilson coefficient is suppressed by $|\mu|^{-2}$ and thus subdominant compared to the contribution of $\mathcal{O}_{1}^{(5)}$. Moreover, the terms in the second line could also cancel with each other to great extent if $M_{1} \simeq M_{2}$. This results in a further suppression of this contribution. Besides, quark-squark loop processes can generate the operator $\mathcal{O}_{2}^{(5)}$ at one-loop level. Their contribution is suppressed by a factor of $m_{\tilde{q}}^{-2}$ on top of a loop factor, with $m_{\widetilde{q}}$ the mass of the squark running in the loop, and thus again subdominant.

EW broken phase. After the Higgs field acquires a vacuum expectation value (VEV), the operators $\mathcal{O}_{1}^{(5)}, \mathcal{Q}_{1}$, and $\mathcal{Q}_{2}$ reduce to the mass terms for bino and wino. The mass matrix for the neutral sector is given by

$$
\mathcal{L}_{\text {mass }}=-\frac{1}{2}\left(\widetilde{B} \widetilde{W}^{0}\right) \mathcal{M}\left(\frac{\widetilde{B}}{\widetilde{W}^{0}}\right)
$$

with

$$
\mathcal{M}=\left(\begin{array}{cc}
M_{1}-\frac{v^{2}}{2} \widetilde{C}_{1} & \frac{v^{2}}{4} C_{1}^{(5)} \\
\frac{v^{2}}{4} C_{1}^{(5)} & M_{2}-\frac{v^{2}}{2} \widetilde{C}_{2}
\end{array}\right) \equiv\left(\begin{array}{cc}
\mathcal{M}_{11} & \mathcal{M}_{12} \\
\mathcal{M}_{12} & \mathcal{M}_{22}
\end{array}\right),
$$

where $v \simeq 246 \mathrm{GeV}$ is the Higgs VEV. This mass matrix can be diagonalized with a $2 \times 2$ unitary matrix $U$, which we parametrize by

$$
U=\left(\begin{array}{cc}
e^{i \alpha} & 0 \\
0 & e^{i \beta}
\end{array}\right)\left(\begin{array}{cc}
\cos \theta & e^{-i \phi} \sin \theta \\
-e^{i \phi} \sin \theta & \cos \theta
\end{array}\right)
$$


Then, we find that the matrix $M$ is diagonalized as $[65,66]$

$$
U^{*} \mathcal{M} U^{\dagger}=\left(\begin{array}{cc}
m_{1} & 0 \\
0 & m_{2}
\end{array}\right),
$$

where $m_{1}$ and $m_{2}$ are real and non-negative, whose values are given by

$m_{1,2}^{2}=\frac{1}{2}\left[\left|\mathcal{M}_{11}\right|^{2}+\left|\mathcal{M}_{22}\right|^{2}+2\left|\mathcal{M}_{12}\right|^{2} \mp \sqrt{\left(\left|\mathcal{M}_{11}\right|^{2}-\left|\mathcal{M}_{22}\right|^{2}\right)^{2}+4\left|\mathcal{M}_{11}^{*} \mathcal{M}_{12}+\mathcal{M}_{22} \mathcal{M}_{12}^{*}\right|^{2}}\right]$.

The mixing angle $\theta$ in the unitary matrix $U$ is given by

$$
\tan \theta=\frac{\left|\mathcal{M}_{11}\right|^{2}-\left|\mathcal{M}_{22}\right|^{2}+\sqrt{\left(\left|\mathcal{M}_{11}\right|^{2}-\left|\mathcal{M}_{22}\right|^{2}\right)^{2}+4\left|\mathcal{M}_{11}^{*} \mathcal{M}_{12}+\mathcal{M}_{22} \mathcal{M}_{12}^{*}\right|^{2}}}{2\left|\mathcal{M}_{11}^{*} \mathcal{M}_{12}+\mathcal{M}_{22} \mathcal{M}_{12}^{*}\right|}
$$

while its phase factors are

$$
\begin{aligned}
e^{i \phi} & =\frac{\mathcal{M}_{11}^{*} \mathcal{M}_{12}+\mathcal{M}_{22} \mathcal{M}_{12}^{*}}{\left|\mathcal{M}_{11}^{*} \mathcal{M}_{12}+\mathcal{M}_{22} \mathcal{M}_{12}^{*}\right|}, \\
\alpha & =\frac{1}{2} \arg \left(\mathcal{M}_{11}-\mathcal{M}_{12} e^{-i \phi} \tan \theta\right), \\
\beta & =\frac{1}{2} \arg \left(\mathcal{M}_{22}+\mathcal{M}_{12} e^{i \phi} \tan \theta\right) .
\end{aligned}
$$

In this calculation, we have implicitly assumed that $\left|\mathcal{M}_{11}\right| \leq\left|\mathcal{M}_{22}\right|$. In terms of the gaugino masses and the Wilson coefficients, these parameters are approximately given as

$$
\begin{aligned}
m_{1}^{2} & \simeq\left|M_{1}\right|^{2}-v^{2} \operatorname{Re}\left(M_{1} \widetilde{C}_{1}^{*}\right), \\
m_{2}^{2} & \simeq\left|M_{2}\right|^{2}-v^{2} \operatorname{Re}\left(M_{2} \widetilde{C}_{2}^{*}\right), \\
\tan \theta & \simeq \frac{v^{2}}{4} \frac{\left|M_{1}^{*} C_{1}^{(5)}+M_{2} C_{1}^{(5) *}\right|}{\left|M_{2}\right|^{2}-\left|M_{1}\right|^{2}}, \\
\phi & \simeq \arg \left(M_{1}^{*} C_{1}^{(5)}+M_{2} C_{1}^{(5) *}\right), \\
\alpha & \simeq \frac{1}{2} \arg \left(M_{1}\right)-\frac{v^{2}}{4} \operatorname{Im}\left(\frac{\widetilde{C}_{1}}{M_{1}}\right), \\
\beta & \simeq \frac{1}{2} \arg \left(M_{2}\right)-\frac{v^{2}}{4} \operatorname{Im}\left(\frac{\widetilde{C}_{2}}{M_{2}}\right) .
\end{aligned}
$$

If $\left|M_{2}\right|-\left|M_{1}\right|$ is $\mathcal{O}(10) \mathrm{GeV}$, which is motivated by the bino-wino coannihilation scenario as shown in refs. [57-60], then the above approximations are valid when $|\mu| \gtrsim \mathcal{O}(10) \mathrm{TeV}$, with which the bino-wino mixing angle is sufficiently small: $\tan \theta \ll 1$. The mass eigenstates are related to the weak eigenstates through the unitary matrix $U$ by

$$
\left(\begin{array}{c}
\widetilde{\chi}_{1}^{0} \\
\widetilde{\chi}_{2}^{0}
\end{array}\right)=U\left(\begin{array}{c}
\widetilde{B} \\
\widetilde{W}^{0}
\end{array}\right) .
$$

In this paper, we assume the bino-like state $\widetilde{\chi}_{1}^{0}$ is slightly lighter than the neutral wino-like state $\widetilde{\chi}_{2}^{0}: m_{1} \lesssim m_{2}$ with the mass difference $\Delta M \equiv m_{2}-m_{1}$ being $\mathcal{O}(10) \mathrm{GeV}$. 
Next, we consider chargino $\tilde{\chi}^{+}$, which is related to the weak eigenstate by

$$
\widetilde{\chi}^{+}=\frac{e^{i \gamma}}{\sqrt{2}}\left(\widetilde{W}^{1}-i \widetilde{W}^{2}\right)
$$

Its mass eigenvalue and phase factor $\gamma$ are given by

$$
m_{\widetilde{\chi}^{+}}=\left|M_{2}-\frac{v^{2}}{2} \widetilde{C}_{2}\right|, \quad \gamma \simeq \beta
$$

On top of that, EW loop corrections make the chargino heavier than the neutral wino by a small amount; this contribution to the mass splitting is evaluated as $\Delta M_{\mathrm{EW}} \simeq 160 \mathrm{MeV}$ at two-loop level $[67,68]$. Note that up to dimension six the higher-dimensional operators do not generate the mass difference between the neutral and charged winos, as we have seen from eq. (2.24) and eq. (2.27). For this reason, although the EW correction $\Delta M_{\mathrm{EW}}$ is quite small, it turns out to be the dominant contribution to the mass difference as long as $|\mu| \gtrsim 10 \mathrm{TeV}$.

Now we summarize the interactions relevant to the decay of $\widetilde{\chi}_{2}^{0}$ and $\tilde{\chi}^{+}$. First, we consider the chargino decay. In this scenario, a chargino $\tilde{\chi}^{+}$mainly decays into $\widetilde{\chi}_{1}^{0}$ because of the degeneracy between $\widetilde{\chi}_{2}^{0}$ and $\tilde{\chi}^{+}$. This decay is caused by the tree-level gauge interactions through the bino-wino mixing. In the mass eigenbasis, the gauge interactions are written as

$$
\mathcal{L}_{\widetilde{\chi}^{0} \widetilde{\chi}^{+} W}=-g \sin \theta \overline{\widetilde{\chi}_{1}^{0}} W^{-}\left[e^{-i(\phi-\alpha+\beta)} P_{L}+e^{i(\phi-\alpha+\beta)} P_{R}\right] \tilde{\chi}^{+}-g \cos \theta \overline{\widetilde{\chi}_{2}^{0}} W^{-} \widetilde{\chi}^{+}+\text {h.c. },
$$

where we have used four-component notation. Notice that these interactions are invariant under the charge conjugation. We use this property below. Using the tree-level relation (2.2), we can obtain an approximate expression for the $\widetilde{\chi}_{1}^{0}-\widetilde{\chi}^{ \pm}-W^{\mp}$ coupling as

$$
-g \sin \theta \simeq-g \sin 2 \beta \frac{m_{W}^{2} \tan \theta_{W}}{\mu \Delta M},
$$

where $\theta_{W}$ is the weak-mixing angle and $m_{W}$ is the $W$-boson mass. We take the gaugino masses to be real in the derivation.

Second, we discuss the decay of heavier neutralino $\widetilde{\chi}_{2}^{0}$ into the lightest neutralino $\widetilde{\chi}_{1}^{0}$. This decay process occurs via the (off-shell) Higgs emission induced by the dimension-five operator $\mathcal{O}_{1}^{(5)}$. The relevant interaction is given by

$$
\mathcal{L}_{\widetilde{\chi}_{1}^{0} \widetilde{\chi}_{2}^{0} h}=-\frac{v}{2} e^{-i(\alpha+\beta)} \cos 2 \theta C_{1}^{(5)} h \widetilde{\chi}_{1}^{0} \widetilde{\chi}_{2}^{0}+\text { h.c. }
$$

Since $\mathcal{O}_{1}^{(5)}$ is of dimension five, this interaction is only suppressed by $|\mu|^{-1}$. Similarly to eq. (2.29), we can approximate the coupling by $-g \tan \theta_{W} \sin (2 \beta) m_{W} / \mu$. Notice that large $\tan \beta$ suppresses this coupling. In this case, the contributions suppressed by $|\mu|^{-2}$ originating from the second term in eq. (2.10) may dominate this contribution.

The interactions of $\widetilde{\chi}_{1}^{0}$ and $\widetilde{\chi}_{2}^{0}$ with a photon and a $Z$ boson are, on the other hand, relatively small. At renormalizable level, there is no such a interaction since $Q=Y=$ 


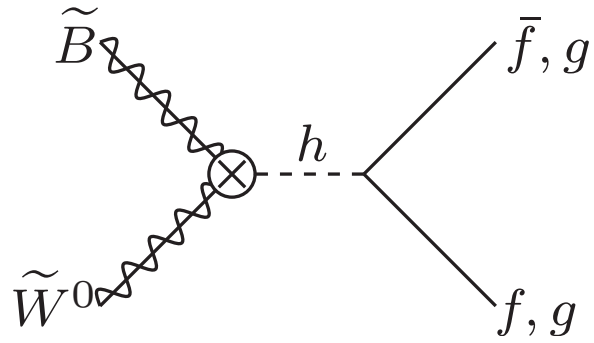

(a) Higgs exchange

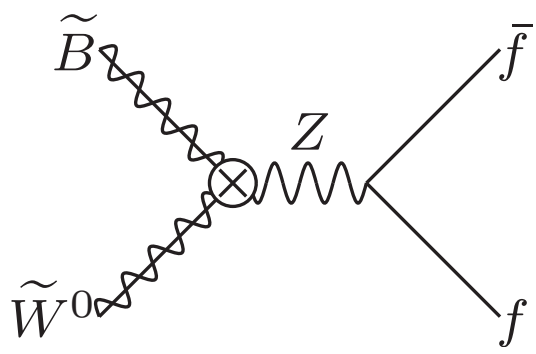

(b) $Z$ boson exchange

Figure 3. Tree-level contributions to the decay of a neutral wino into the bino LSP. The symbols $\bigotimes$ in the diagrams represent the effective interactions, which are suppressed by powers of the inverse of the Higgsino mass $\mu$.

$T_{3}=0$ for both of these particles. As discussed above, the contribution of the dimensionfive operator $\mathcal{O}_{2}^{(5)}$ is suppressed by $|\mu|^{-2}$ besides the one loop factor. The dimension-six operator $\mathcal{O}^{(6)}$ also gives rise to such interactions at tree level,

$$
\mathcal{L}_{\widetilde{\chi}_{1}^{0} \widetilde{\chi}_{2}^{0} Z}=\frac{g_{Z} v^{2}}{4} e^{i(\alpha-\beta)} C^{(6)} \widetilde{\chi}_{1}^{0 \dagger} \bar{\sigma}^{\mu} \widetilde{\chi}_{2}^{0} Z_{\mu}+\text { h.c. },
$$

where $g_{Z} \equiv \sqrt{g^{\prime 2}+g^{2}}$. This interaction is again suppressed by $\cos (2 \beta)|\mu|^{-2}$. Consequently, compared to the Higgs interaction in eq. (2.30), the interactions with a photon and a $Z$ boson are fairly small if we take $|\mu|$ to be sufficiently large.

An important caveat here is that these interactions could be important if $\tan \beta$ is large, since the Higgs coupling in eq. (2.30) is highly suppressed in this case as mentioned above. Although the mini-split type models favor small $\tan \beta$ to explain the $125 \mathrm{GeV}$ Higgs mass, a moderate size of $\tan \beta$ may be allowed if stop masses are rather light. In such cases, the terms suppressed by $|\mu|^{-2}$ can also be significant.

\subsection{Decay length in bino-wino coannihilation}

Taking the above discussion into account, we now estimate the decay length and branching ratios of the neutral wino-like state $\widetilde{\chi}_{2}^{0}$. As we have seen above, when $|\mu|$ is large enough, the Higgs interaction (2.30) dominates the others. This interaction gives rise to threebody decay processes shown in figure 3 a when the mass difference between $\widetilde{\chi}_{1}^{0}$ and $\widetilde{\chi}_{2}^{0}$, $\Delta M$, is as small as $\mathcal{O}(10) \mathrm{GeV}$. In this case, the dominant decay channel is $\widetilde{\chi}_{2}^{0} \rightarrow \widetilde{\chi}_{1}^{0} b \bar{b}$, whose decay amplitude is suppressed by the bottom-quark Yukawa coupling in addition to the three-body phase space factor and $|\mu|^{-1}$. Moreover, the decay rate is kinematically suppressed due to the small mass difference $\Delta M$. For these reasons, the resultant decay rate is considerably small and $\widetilde{\chi}_{2}^{0}$ has a sizable decay length, as we see below.

Notice that, as we mentioned above, tree-level sfermion exchange contributions to the three-body decay processes are generically much smaller than the Higgs exchange contribution. The former contributions can be expressed by dimension-six effective operators whose coefficients are $\sim g g^{\prime} / \widetilde{m}^{2}$. On the other hand, after integrating out the Higgs boson, the diagram in figure 3 a yields a dimension-six operator with its coefficient being 


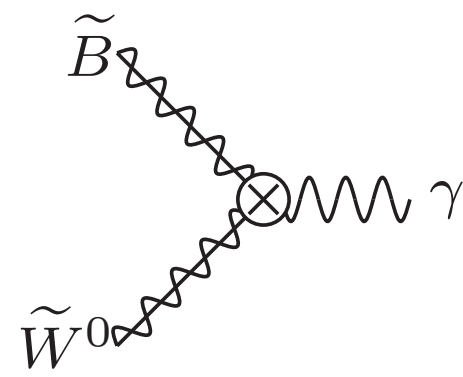

(a) Dipole-type contribution

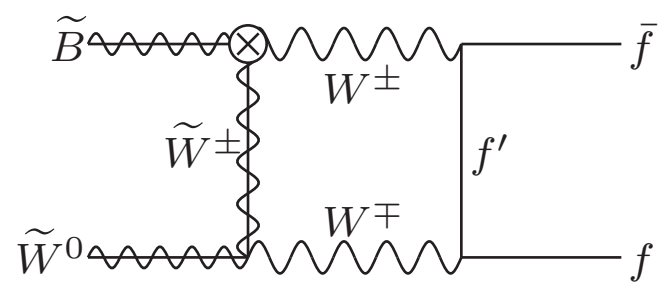

(b) Box diagram

Figure 4. One-loop contributions to the decay of a neutral wino into the bino LSP. The symbols $\bigotimes$ in the diagrams represent the effective interactions, which are suppressed by powers of the inverse of the Higgsino mass $\mu$.

$\sim g g^{\prime}(\sin 2 \beta) m_{b} /\left(\mu m_{h}^{2}\right)$. Therefore, the Higgs exchange contribution dominates sfermion ones as long as $|\mu| \ll \mathcal{O}\left(10^{3}\right) \mathrm{TeV} \times \sin 2 \beta \cdot\left(\widetilde{m} / 10^{2} \mathrm{TeV}\right)^{2}$.

At tree-level, we also have the virtual $Z$ exchange contribution induced by the interaction (2.31), which is illustrated in figure 3b. As discussed above, the interaction (2.31) is suppressed by a factor of $|\mu|^{-2}$, and thus their contribution is subdominant. Notice that ordinary strategies on the searches for charginos and neutralinos at the LHC rely on the leptonic decay channel $\widetilde{\chi}_{2}^{0} \rightarrow \widetilde{\chi}_{1}^{0} \ell^{+} \ell^{-}$induced by this contribution $[69,70]$. Since the decay branch into the channel is extremely suppressed in our scenario, we need an alternative way to probe the bino-wino coannihilation region at the LHC. This is the subject of the next section.

The two-body decay process $\widetilde{\chi}_{2}^{0} \rightarrow \widetilde{\chi}_{1}^{0} \gamma$ shown in figure 4a is induced by the dipole-type operator $\mathcal{O}_{2}^{(5)}$, which is generated at one-loop level. As discussed above, this contribution is also suppressed by $|\mu|^{-2}$. As a result, it turns out that the three-body decay processes in figure 3a dominate this two-body process in most of parameter space we are interested in. One may think that the gauge interactions (2.28) also induces the process in figure 4a via the chargino- $W$ boson loop diagram. We find, however, that this contribution vanishes. To see the reason, notice that the amplitude of the diagram represented in figure 4a is odd under the charge conjugation $C$, as $\widetilde{\chi}_{1}^{0}$ and $\widetilde{\chi}_{2}^{0}$ are Majorana fields and a photon is $C$-odd. On the other hand, as noted above, the gauge interactions in eq. (2.28) preserve the $C$ symmetry. The electromagnetic interaction is also invariant under the charge conjugation. Then, it follows that any amplitude induced by these interactions should be $C$-even, hence their contribution to the process in figure 4 a vanishes. After all, the $\widetilde{\chi}_{2}^{0} \rightarrow \widetilde{\chi}_{1}^{0} \gamma$ decay is subdominant as long as $\left|M_{1}\right|,\left|M_{2}\right|, \ll|\mu|$, and therefore we focus on the tree-level processes in figure 3 in the following analysis.

However, there are several possibilities in which the above conclusion should be altered. Firstly, if the Higgsino mass is rather small, the contribution of the dipole operator $\mathcal{O}_{2}^{(5)}$ can be significant. Indeed, such a situation may be realized in the framework of spread SUSY, as discussed in refs. [13, 71, 72]. If the decay branch into the $\widetilde{\chi}_{2}^{0} \rightarrow \widetilde{\chi}_{1}^{0} \gamma$ channel is sizable, the collider signature discussed in the subsequent section would be modified. Secondly, as already noted above, the Higgs exchange contribution decreases if $\tan \beta$ is large. Such 


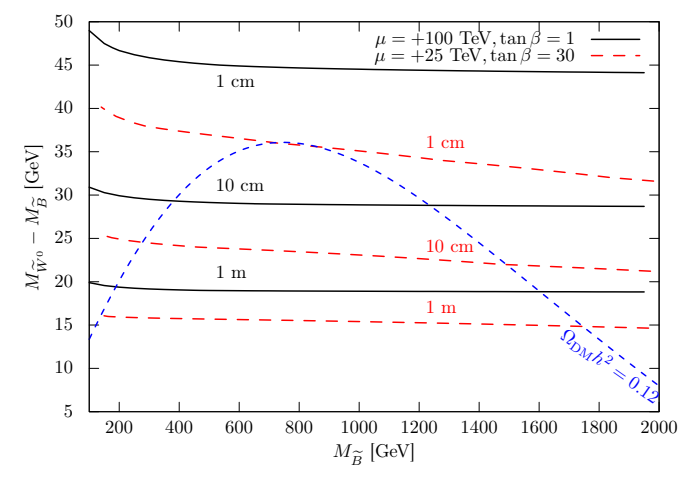

(a) $c \tau_{\widetilde{W}^{0}}$ in $M_{\widetilde{B}^{-}}-\Delta M$ plane.

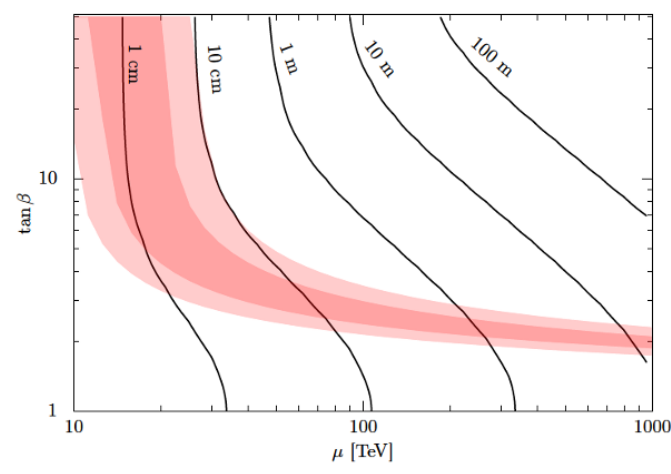

(b) $c \tau_{\widetilde{W}^{0}}$ in $\mu-\tan \beta$ plane.

Figure 5. Decay length $c \tau_{\widetilde{W}^{0}}$ of a neutral wino $\widetilde{\chi}_{2}^{0}$. (a): black solid (red dashed) lines show contours for the decay length of the neutral wino for $\mu=+100 \mathrm{TeV}$ and $\tan \beta=1(\mu=+25 \mathrm{TeV}$ and $\tan \beta=30)$. Blue dashed line corresponds to the parameter region which realizes $\Omega_{\mathrm{DM}} h^{2}=0.12$. (b): decay length of the $430 \mathrm{GeV}$ neutral wino into the $400 \mathrm{GeV}$ bino LSP. The dark (light) red area shows the $1 \sigma(2 \sigma)$ preferred parameter region to explain the $125 \mathrm{GeV}$ Higgs mass. Here we set the gluino mass to be $1.5 \mathrm{TeV}$ and the sfermion mass scale to be $\widetilde{m}=\mu$.

a situation may occur if the SUSY breaking scale is as low as $\mathcal{O}(10) \mathrm{TeV}$. Again, in this case, the decay branching ratios may change considerably. Thirdly, if the mass difference $\Delta M$ is smaller than $\mathcal{O}(10) \mathrm{GeV}$, then the $\widetilde{\chi}_{2}^{0} \rightarrow \widetilde{\chi}_{1}^{0} b \bar{b}$ decay mode is highly suppressed, and other decay modes can be significant, such as one-loop diagrams shown in figure $4 \mathrm{~b}$. In addition, the two-body decay process containing a bottomonium induced by the virtual Higgs exchange may also be important in such a situation. Since these possibilities are only relevant to rather specific parameter region in our setup, we do not consider them in this paper, and discuss these contributions on another occasion.

In summary, the mass spectrum for EW gauginos and the suppression factor in their decay rates are illustrated in figure 1 . We consider a specific collider signature for this mass spectrum in the following section.

Now we evaluate the decay length $c \tau_{\widetilde{W}^{0}}$ of the neutral wino-like state $\widetilde{\chi}_{2}^{0}$. In figure $5 \mathrm{a}$, we plot it in the $M_{\widetilde{B}^{-}}-\Delta M$ plane. Here, we set $\mu=+100 \mathrm{TeV}(+25 \mathrm{TeV})$ and $\tan \beta=1(30)$ in the black solid (red dashed) lines. The gaugino masses are taken to be real and positive in this figure. The blue dashed line corresponds to the parameter region where the thermal relic abundance of the bino LSP agrees to the observed DM density $\Omega_{\mathrm{DM}} h^{2}=0.12$. As can be seen from this figure, a neutral wino has a sizable decay length in a wide range of parameter region motivated by the bino-wino coannihilation scenario. This observation is a key ingredient for the search strategy discussed in the next section. In figure 5b, on the other hand, the decay length $c \tau_{W^{0}}$ is plotted in the $\mu$-tan $\beta$ plane. Here, we set $M_{1}=+400 \mathrm{GeV}$ and $M_{2}=+430 \mathrm{GeV}$. For reference, we also show the $1 \sigma(2 \sigma)$ preferred region for the $125 \mathrm{GeV}$ Higgs mass in the dark (light) red shaded area, where the gluino mass is set to be $1.5 \mathrm{TeV}$ and the scalar mass scale is taken to be equal to the Higgsino mass, $\widetilde{m}=\mu$. We find that the decay length grows as $\tan \beta$ is taken to be large. This is because the Higgs interaction (2.30) is suppressed in this case. Anyway, a neutral wino has 
a decay length of $\gtrsim 1 \mathrm{~cm}$ over the parameter region in this figure, which could be observed at the LHC as we see in the next section.

Before closing this section, let us comment on the bino-wino coannihilation. One may wonder the chemical equilibrium between the wino and bino, which is essential for the successful coannihilation, can be kept even if $\mu$ is significantly large. Unlike the bino-gluino coannihilation case $[56,73]$, the bino and wino can interact with each other through the dimension-five operators. Thus, the interchange of $\widetilde{B} \leftrightarrow \widetilde{W}$ at the freeze-out temperature is rapid enough for the chemical equilibrium between them to be maintained, even though $\mu$ is at $\mathrm{PeV}$ scale.

\section{LHC search}

We have seen in the previous section that a neutral wino in our scenario has a sizable decay length. In this section, we discuss the prospects of the searches for such a long-lived wino at the LHC. A decay process of a neutral wino with $c \tau_{\widetilde{W}^{0}} \gtrsim 1 \mathrm{~mm}$ can be observed as a DV plus missing transverse energy event. The ATLAS collaboration has reported the result of searches for such events by using the $20.3 \mathrm{fb}^{-1}$ data set collected at the LHC with a center-of-mass energy of $\sqrt{s}=8 \mathrm{TeV}$ [74]. In section 3.1, we begin with reviewing this ATLAS result for the DV search. It turns out, however, that the existing result cannot be directly applied to the bino-wino coannihilation scenario, since the ATLAS study mainly focus on high-mass DVs. Thus, we need to extend their analysis so that a smaller mass DV can also be probed. We describe our setup in section 3.2. Finally, in section 3.3, we show the prospects for the long-lived wino search in the cases of both direct and gluino mediated productions.

\subsection{Previous LHC study}

The LHC finished the operation at center of mass energy of $8 \mathrm{TeV}$ in 2012 . The ATLAS and the CMS experiments explored many types of neutral long-lived SUSY particles so far, which are listed as follows [74-83]:

- Long-lived gluino in the mini-split SUSY which decays into two quarks and a neutralino.

- Long-lived neutralino in the $R$-parity violation scenario which decays into leptons and quarks.

- Long-lived neutralino in the gauge mediation scenario which decays into a photon or $Z$ boson, and a gravitino.

In these searches, the decay products from the long-lived particles have large impact parameters, which are distinct from background events that originate from the primary vertex. Our search strategy proposed in this paper mainly considers signals including two quarks from a DV, and thus the long-lived gluino search in the mini-split SUSY performed by the ATLAS experiment [74] would be a good reference. 
The ATLAS study [74] employs several triggers for long-lived particles. Among them, the trigger utilizing missing transverse energy $\left(E_{\mathrm{T}}^{\mathrm{miss}}\right)$ has the best sensitivity for a longlived gluino; $E_{\mathrm{T}}^{\text {miss }}>80 \mathrm{GeV}$ is required for the trigger, $E_{\mathrm{T}}^{\text {miss }}>100 \mathrm{GeV}$ by offline filters, and eventually $E_{\mathrm{T}}^{\text {miss }}>180 \mathrm{GeV}$ at the offline selection.

Reconstruction of DVs is CPU-intensive due to many tracks with high impact parameters. In order to reduce the computation time, only the events with two "trackless" jets of $P_{\mathrm{T}}>50 \mathrm{GeV}$ are processed. The "trackless" jets are defined such that the scalar sum of the transverse momenta of the tracks in the jet should be less than $5 \mathrm{GeV}$ with the standard track reconstruction. Such jets could be accompanied by decays of long-lived particles which occur away from the primary vertex.

At the end of the selections, a DV with more than four tracks whose invariant mass is more than $10 \mathrm{GeV}$ is treated as a signal event. Here, each track is supposed to have the mass of the charged pion $m_{\pi^{ \pm}}$. After the selections, the reconstruction efficiency of at least a DV from a pair of produced gluinos with a decay length of $c \tau_{\widetilde{g}}=\mathcal{O}(1) \mathrm{mm}$ is almost zero, which increases up to more than $50 \%$ when $c \tau_{\widetilde{g}}=\mathcal{O}(10) \mathrm{mm}$. Here, a gluino mass of $1400 \mathrm{GeV}$ is assumed in the estimation. Beyond the point, the reconstruction efficiency gradually decreases and reaches zero for $c \tau_{\widetilde{g}} \gtrsim \mathcal{O}(1) \mathrm{m}$.

In the bino-wino coannihilation scenario, a favored value of $\Delta M$ is $\lesssim 30 \mathrm{GeV}$, as can be seen from figure $5 \mathrm{a}$. In this case, jets from a decay of a neutral wino are too soft to satisfy the $P_{\mathrm{T}}$ condition of the trackless jets. To probe the scenario with the DV searches, therefore, we need to relax the above requirements to some extent, which we discuss in what follows.

\subsection{Signal simulation setup}

Now we adjust the ATLAS DV search method such that it has sensitivity to long-lived neutral winos, and discuss its prospects at the $14 \mathrm{TeV}$ LHC running. At the LHC, there are two channels to produce a neutral wino. One is its direct production and the other is gluino mediated production in which a produced gluino decays into a neutral wino. In both cases, the $E_{\mathrm{T}}^{\text {miss }}$ trigger is the most efficient among the DV search triggers, just like the long-lived gluino search. In this study, we adopt $E_{\mathrm{T}}^{\text {miss }}>100 \mathrm{GeV}$ and $200 \mathrm{GeV}$ for the $8 \mathrm{TeV}$ and $14 \mathrm{TeV}$ LHC cases, respectively. As for the DV detection criteria, we assume the same setup as the ATLAS study:

- The number $N_{\text {tr }}$ of charged tracks forming a DV should be grater than four, where each track should have a transverse momentum of $P_{\mathrm{T}}>1 \mathrm{GeV}$.

- The invariant mass of the sum of the momenta of the tracks, $m_{\mathrm{DV}}$, should be greater than $10 \mathrm{GeV}$, with the mass of each track being assumed to be $m_{\pi^{ \pm}}$.

Notice that we have dropped the $P_{\mathrm{T}}$ condition for the trackless jets adopted in the case of the long-lived gluino search.

To see expected size of $m_{\mathrm{DV}}$, in figure $6 \mathrm{a}$, we show the distributions of $m_{\mathrm{DV}}$. Here we consider the wino direct production case, with setting the wino and bino masses to be $M_{\widetilde{W}}=400 \mathrm{GeV}$ and $M_{\widetilde{B}}=370 \mathrm{GeV}$, respectively. A produced neutral wino is assumed to 


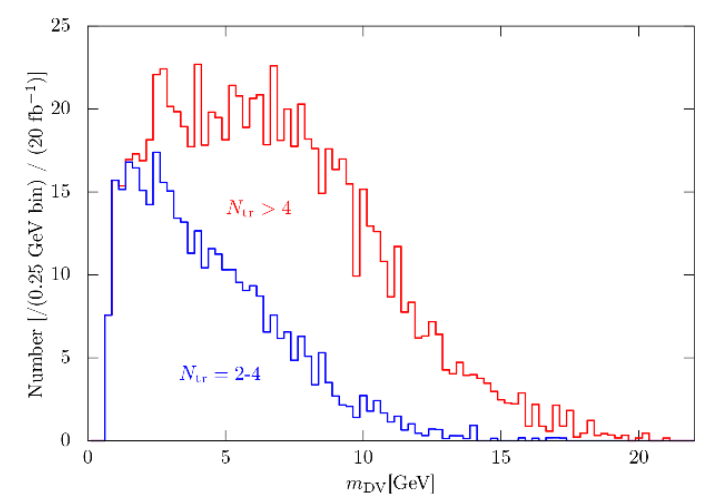

(a) Distributions of $m_{\mathrm{DV}}$

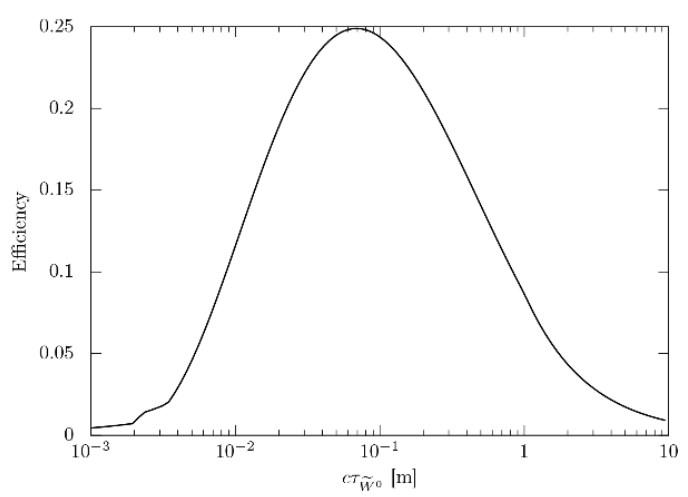

(b) Single DV reconstruction efficiency

Figure 6. (a) Distributions of $m_{\mathrm{DV}}$. Here, we set $M_{\widetilde{W}}=400 \mathrm{GeV}$ and $M_{\widetilde{B}}=370 \mathrm{GeV}$. Winos are produced directly, which are assumed to decay through the Higgs exchange process. (b) Single DV reconstruction efficiency as a function of the neutral wino decay length.

decay into a bino via the Higgs exchange process. As can be seen, $m_{\mathrm{DV}}$ is expected to be $\sim 10 \mathrm{GeV}$, if $\Delta M=30 \mathrm{GeV}$, and a sizable number of events provide $m_{\mathrm{DV}}>10 \mathrm{GeV}$.

We also need the DV detection efficiency to estimate the number of signals. We estimate it from the result of the ATLAS gluino search [74]. Figure 19 of ref. [74] shows a lower-bound on the gluino mass as a function of the gluino decay length $c \tau_{\widetilde{g}}$, with the LSP mass of $100 \mathrm{GeV}$ being assumed. In this case, the $\mathrm{DV}+E_{\mathrm{T}}^{\text {miss }}$ search provides the strongest constraints in most of the parameter region. The mass difference between the gluino and the LSP is typically greater than $1 \mathrm{TeV}$ in this plot. Since the transverse momenta of jets and the LSP produced by the gluino decay are large, acceptance rates for the trigger and the event filter are expected to be almost $100 \%$. Moreover, the acceptance rate of the truthlevel events for the requirements of the DV criteria and the trackless jets is also expected to be almost $100 \%$. As a consequence, we can estimate the decay-length dependent detection efficiency of DVs, $\epsilon_{\widetilde{g}}$, as

$$
\epsilon_{\widetilde{g}}(c \tau)=\frac{\sigma_{\mathrm{obs}}}{\sigma(p p \rightarrow \widetilde{g} \widetilde{g})}
$$

where $\sigma_{\text {obs }}(=0.15 \mathrm{fb})$ is observed upper-bound on the cross section of the $\mathrm{DV}+E_{\mathrm{T}}^{\text {miss }}$ events [74]. In this case, for each long-lived gluino DV event, the number of DVs is two. Therefore, the reconstruction efficiency of a single DV, $\epsilon_{\mathrm{DV}}$, is about a half of $\epsilon_{\tilde{g}}$. For $c \tau_{\widetilde{g}} \gtrsim 1 \mathrm{~m}$, the DV reconstruction efficiency would be inversely proportional to decay length, since gluinos must decay within the tracker system. Although figure 19 of ref. [74] only shows the case of $1<c \tau_{\widetilde{g}}<1000 \mathrm{~mm}$, we extrapolate the efficiency up to $c \tau_{\widetilde{g}}>1 \mathrm{~m}$ taking into account a suppression factor of $\propto\left(c \tau_{\widetilde{g}}\right)^{-1}$. In figure 6b, we show the estimated efficiency $\epsilon_{\mathrm{DV}}$ as a function of the decay length of the neutral wino. This figure shows that the DV reconstruction efficiency is sizable for $1 \mathrm{~cm} \lesssim c \tau_{\widetilde{W}^{0}} \lesssim 1 \mathrm{~m}$, which is maximized when $c \tau_{\widetilde{W}^{0}} \sim 10 \mathrm{~cm}$. This estimation assumes the velocity distribution of the long-lived winos to be the same as that of long-lived gluinos in ref. [74]. Although these two distributions are potentially different, we do not expect that this difference alters our results drastically. 
The total acceptance rate is the product of the efficiency and the rate of passing the above $E_{\mathrm{T}}^{\text {miss }}$ triggers and the DV criteria. To estimate the acceptance rate, we use the program packages Madgraph5 [84], Pythia6 [85], and Delphes3 [86], while for the cross sections of the SUSY particles we use PRosPINO2 [87].

Note that the estimation of the prospects discussed below possibly suffers from large uncertainties. At first, the DV detection efficiency depends on the momentum distributions of the particles which generate DVs. The small mass difference $\Delta M$ reduces the number of tracks and makes their momenta lower, which may impair the DV reconstruction efficiency. On the other hand, the current DV criteria is designed for the high-mass DVs, and not optimized for the low-mass DVs like the present wino case. A small modification in the DV criteria can drastically change the acceptance rate as seen in figure $6 \mathrm{a}$ and can potentially improve the detection efficiency. Moreover, although the present DV reconstruction efficiency is obtained for DVs associated with light-quark jets, we assume the neutral wino mainly decays into $b$ jets in the following analysis. With the present ATLAS analysis, the reconstruction efficiency of a DV accompanied with $b$ jets is expected to be worse than those with light quarks. However, the neutral wino decaying into two $b$ quarks provides characteristic signatures compared to light-quark decays; namely, the $b$ quarks cause additional DVs originating from a DV given by a decay of a long-lived neutral wino. This specific signature may give further optimization of the search of the wino DV and improve the reconstruction efficiency in the future LHC experiments. For more precise estimation, we need full detector simulation and this is out of the scope of this paper. We use the above simplified setup to see the prospects qualitatively in what follows.

\subsection{LHC prospects}

First, let us discuss the case where winos are directly produced. The crucial difference between the ATLAS gluino search and the present direct wino search is the sizes of the missing transverse momentum and the invariant mass of the tracks from DVs, due to the small mass difference between the wino and bino. Both factors reduce the signal acceptance of the wino processes. The missing energy mainly comes from the back reaction of the initial state radiations in the wino production. For $M_{\widetilde{W}}=400 \mathrm{GeV}$ and $\Delta M=30 \mathrm{GeV}$, the acceptance rates for the missing energy $\left(E_{\mathrm{T}}^{\text {miss }}>100 \mathrm{GeV}\right.$ for the $8 \mathrm{TeV}$ running and $200 \mathrm{GeV}$ for $14 \mathrm{TeV}$ ) and DV are about $3 \%$ and $1 \%$, respectively. Here, we assume the neutral wino decays into a pair of bottom quarks and a bino via the Higgs boson exchange process.

In figure 7a, we show the prospects for the long-lived wino search at the LHC. Here we assume zero background and require three signal events. The mass difference $\Delta M$ is set to be $30 \mathrm{GeV}$. The red and blue solid lines show the prospects for $8 \mathrm{TeV}$ and $14 \mathrm{TeV} \mathrm{LHC}$ run, respectively. Note that this estimation is based on our simplified method described in the previous subsection. The DV reconstruct efficiency in the real detector could be different, since the DV masses in the current case are much smaller than those expected for the ATLAS model points [74]. Moreover, $b$ jets from DVs may worsen the efficiency. On the other hand, the current ATLAS analysis is not optimized for the low-mass DV and $b$ jets, and thus future development on search techniques for such DVs may improve 


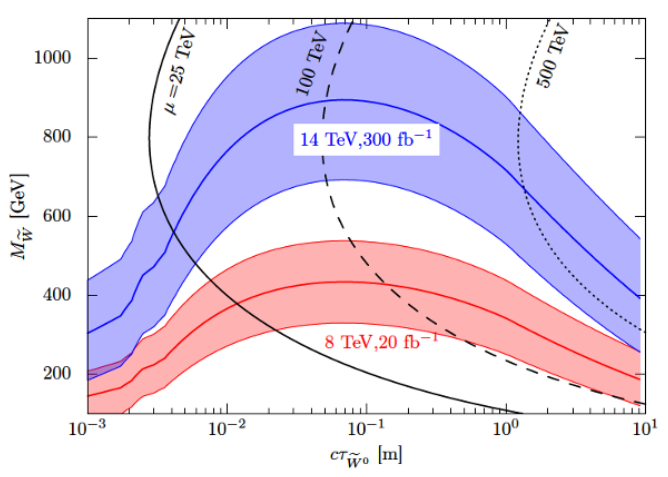

(a) Direct wino production

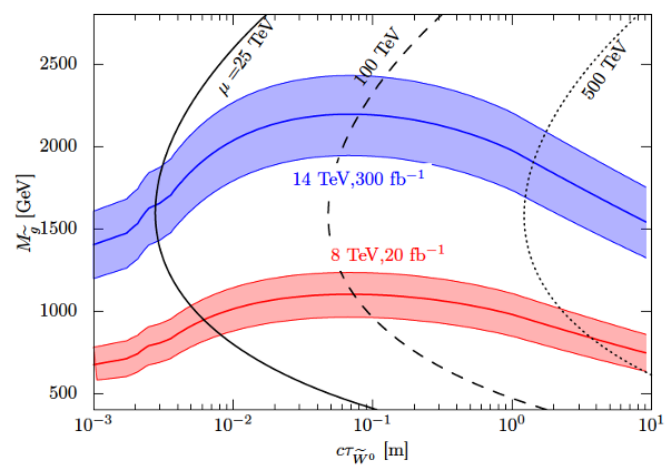

(b) Gluino production

Figure 7. Prospects for the long-lived wino search. (a): direct wino production, (b): gluino production with $M_{\widetilde{g}}=2 M_{\widetilde{W}}$. In both cases, we assume the wino-bino mass difference is $30 \mathrm{GeV}$. Black solid, dashed, and dotted lines show contours corresponding to $\mu=25$, 100, and $500 \mathrm{TeV}$, respectively, with $\tan \beta=2$ and $\Delta M$ taken so that it gives the correct DM abundance.

the efficiency. These possibilities result in large uncertainties in the present estimation. Here, we estimate the uncertainties by scaling the acceptance rate by factors of three and one third, and show them as the bands in the figure. For reference, we also show the decay length of the neutral wino for $\mu=25,100,500 \mathrm{TeV}$ with $\tan \beta=2$ in the black solid, dashed, and dotted lines, respectively, with $\Delta M$ taken so that it realizes the correct DM abundance. From this figure, we find that using the DV searches we may probe a wino with a mass of $400 \mathrm{GeV}(800 \mathrm{GeV})$ at the $8 \mathrm{TeV}(14 \mathrm{TeV})$ LHC if its decay length is $\mathcal{O}(10) \mathrm{cm}$. This should be contrasted with other wino searches which do not use DVs. Without DVs, the discovery of a wino having $\Delta M=\mathcal{O}(10) \mathrm{GeV}$ is extremely difficult; for such a neutral wino decaying into a bino via the Higgs boson exchange, the soft leptons or mono-jet plus missing energy searches at the LHC can provide essentially no constraints on this mass spectrum.

Next, let us discuss the case in which winos are produced from decays of gluinos. If gaugino masses are determined by only the anomaly mediation effects and the threshold effects of the Higgsino loop, the gluino mass is about 2-4 times larger than the wino mass in the case of the bino-wino coannihilation. If the gluino mass is not so large, wino productions through gluino decays are also active. Unlike the direct wino production case, the acceptance rate of the missing energy trigger is close to $100 \%$ as long as the gluino-wino mass difference is sufficiently large. The rate for passing the above DV criteria is around $5 \%$. The gluino decay mode strongly depends on the squark mass sector. Here we assume that left-handed squarks dominantly contribute the gluino decay, and the branching fraction of a gluino decaying into a wino, $\widetilde{g} \rightarrow \widetilde{W}^{0}$, is about $30 \%$. In figure $7 \mathrm{~b}$, we show the prospects for the wino search via this channel. Here, we take $M_{\widetilde{g}}=2 M_{\widetilde{W}}$, and $\Delta M=30 \mathrm{GeV}$. Again, the black solid, dashed, and dotted lines show contours corresponding to $\mu=25$, 100 , and $500 \mathrm{TeV}$, respectively, with $\tan \beta=2$ and $\Delta M$ taken so that it gives the correct $\mathrm{DM}$ abundance. We find that in this case the $14 \mathrm{TeV}$ LHC reach for the gluino mass is as high as $2 \mathrm{TeV}$ when $c \tau_{\widetilde{W}^{0}} \sim 10 \mathrm{~cm}$. 
For the gluino-mediated case, a sizable mass difference between gluino and wino leads to rather high jet activity and large missing energy. Therefore, usual jets plus missing energy search (without DVs) can probe a wide range of gluino masses. For $M_{\widetilde{g}}=2 M_{\widetilde{W}}$, a gluino with a mass of $1 \mathrm{TeV}(2 \mathrm{TeV})$ can be probed with conventional jets plus missing energy search at the LHC8 (14). The gluino search with DVs discussed here may not drastically improve the gluino discovery range, since the DV reconstruction efficiency is quite low. Optimization of the DV criteria may increase this efficiency, though. Even if gluinos are first discovered in the ordinary jets plus missing energy searches, however, it is still important to search for the gluino-mediated wino production events using DVs as well; it is difficult for the former searches to distinguish the bino-wino coannihilation scenario from merely the wino LSP case, while this is possible for the DV searches. In addition, the DV search strategy may allow us to extract the bino-wino mass difference through energy measurements of decay product, which is quite important to test the bino-wino coannihilation scenario.

\section{Conclusion and discussion}

In this paper, we study the neutral wino decay in the bino-wino coannihilation scenario and discuss its collider signature. We find that the neutral wino has a considerably long lifetime in the mini-split spectrum, and is detectable at the LHC by means of the DV searches. To assess the prospects for the detectability, we study the direct and gluinomediated productions of the neutral wino at the 8 and $14 \mathrm{TeV}$ LHC running. It turns out that winos (gluinos) with a mass of $800 \mathrm{GeV}(2 \mathrm{TeV})$ can be probed at the next stage of the LHC in the former (latter) production case. The search for the directly produced winos with DVs is very powerful, compared to the conventional searches. For the gluino, this is not so efficient and comparable to the conventional jets plus missing energy searches. However, note that this conclusion can be altered since our estimation of the detection rate has potentially large uncertainty. A more realistic detector simulation is needed to obtain robust prospects.

In this paper, we focus on the parameter region where the tree-level contributions are dominant. However, as discussed above, higher loop processes may significantly contribute to the wino decay in certain parameter region: a moderate $\mu$ case $|\mu| \lesssim \mathcal{O}(10) \mathrm{TeV}$, a highdegeneracy mass case $\Delta M \sim \mathcal{O}(1) \mathrm{GeV}$, and so on. In these cases, the decay branches of wino are altered, accordingly we may need to modify our collider search strategy. Detailed study with a more realistic detector simulation and more accurate evaluation of the wino decay will be done elsewhere.

If the Higgsino mass is rather small, then the DV search strategy discussed in this paper does not work as the neutral wino decay length is too short, $c \tau_{\widetilde{W}^{0}}<1 \mathrm{~mm}$. However, it is possible to probe such a region with low-energy precision experiments like the DM direct detection experiments and the measurements of electric dipole moments $[72,88,89]$. The interplay between the DV search and these experiments will also be discussed on another occasion. 
Lastly let us comment on the prospects of future lepton colliders. The high-energy lepton collider is very powerful tool to probe directly or indirectly the wino sector [90, 91]. However it is non-trivial whether the lepton collider can observe the wino DV signal, since the neutral wino production cross section is quite small. Still, neutral winos may be produced through a loop process or multi-particle production channel. In contrast, they can be considerably produced both directly and indirectly at the LHC. In this sense, the LHC experiment is quite suitable for the DV wino search.

\section{Acknowledgments}

We thank Kazuki Sakurai for informing us of his related work in ref. [92]. The work of N.N. is supported by Research Fellowships of the Japan Society for the Promotion of Science for Young Scientists.

Open Access. This article is distributed under the terms of the Creative Commons Attribution License (CC-BY 4.0), which permits any use, distribution and reproduction in any medium, provided the original author(s) and source are credited.

\section{References}

[1] J.D. Wells, Implications of supersymmetry breaking with a little hierarchy between gauginos and scalars, hep-ph/0306127 [INSPIRE].

[2] J.D. Wells, PeV-scale supersymmetry, Phys. Rev. D 71 (2005) 015013 [hep-ph/0411041] [INSPIRE].

[3] N. Arkani-Hamed and S. Dimopoulos, Supersymmetric unification without low energy supersymmetry and signatures for fine-tuning at the LHC, JHEP 06 (2005) 073 [hep-th/0405159] [INSPIRE].

[4] G.F. Giudice and A. Romanino, Split supersymmetry, Nucl. Phys. B 699 (2004) 65 [Erratum ibid. B 706 (2005) 65] [hep-ph/0406088] [INSPIRE].

[5] N. Arkani-Hamed, S. Dimopoulos, G.F. Giudice and A. Romanino, Aspects of split supersymmetry, Nucl. Phys. B 709 (2005) 3 [hep-ph/0409232] [INSPIRE].

[6] N. Arkani-Hamed, S. Dimopoulos and S. Kachru, Predictive landscapes and new physics at a TeV, hep-th/0501082 [INSPIRE].

[7] F. Gabbiani, E. Gabrielli, A. Masiero and L. Silvestrini, A Complete analysis of FCNC and CP constraints in general SUSY extensions of the standard model, Nucl. Phys. B 477 (1996) 321 [hep-ph/9604387] [inSPIRE].

[8] T. Goto and T. Nihei, Effect of RRRR dimension five operator on the proton decay in the minimal SU(5) SUGRA GUT model, Phys. Rev. D 59 (1999) 115009 [hep-ph/9808255] [INSPIRE].

[9] H. Murayama and A. Pierce, Not even decoupling can save minimal supersymmetric SU(5), Phys. Rev. D 65 (2002) 055009 [hep-ph/0108104] [INSPIRE].

[10] ATLAS collaboration, Observation of a new particle in the search for the Standard Model Higgs boson with the ATLAS detector at the LHC, Phys. Lett. B 716 (2012) 1 [arXiv: 1207.7214] [INSPIRE]. 
[11] CMS collaboration, Observation of a new boson at a mass of $125 \mathrm{GeV}$ with the CMS experiment at the LHC, Phys. Lett. B 716 (2012) 30 [arXiv:1207.7235] [INSPIRE].

[12] ATLAS, CMS collaborations, Combined Measurement of the Higgs Boson Mass in pp Collisions at $\sqrt{s}=7$ and $8 \mathrm{TeV}$ with the ATLAS and CMS Experiments, Phys. Rev. Lett. 114 (2015) 191803 [arXiv:1503.07589] [INSPIRE].

[13] L.J. Hall and Y. Nomura, Spread Supersymmetry, JHEP 01 (2012) 082 [arXiv:1111.4519] [INSPIRE].

[14] L.J. Hall, Y. Nomura and S. Shirai, Spread Supersymmetry with Wino LSP: Gluino and Dark Matter Signals, JHEP 01 (2013) 036 [arXiv:1210.2395] [INSPIRE].

[15] M. Ibe and T.T. Yanagida, The Lightest Higgs Boson Mass in Pure Gravity Mediation Model, Phys. Lett. B 709 (2014) 374 [arXiv:1112.2462] [INSPIRE].

[16] M. Ibe, S. Matsumoto and T.T. Yanagida, Pure Gravity Mediation with $m_{3 / 2}=10-100 \mathrm{TeV}$, Phys. Rev. D 85 (2012) 095011 [arXiv:1202.2253] [INSPIRE].

[17] A. Arvanitaki, N. Craig, S. Dimopoulos and G. Villadoro, Mini-Split, JHEP 02 (2013) 126 [arXiv: 1210.0555] [INSPIRE].

[18] N. Arkani-Hamed, A. Gupta, D.E. Kaplan, N. Weiner and T. Zorawski, Simply Unnatural Supersymmetry, arXiv:1212.6971 [INSPIRE].

[19] J.L. Evans, M. Ibe, K.A. Olive and T.T. Yanagida, Universality in Pure Gravity Mediation, Eur. Phys. J. C 73 (2013) 2468 [arXiv:1302.5346] [InSPIRE].

[20] Y. Okada, M. Yamaguchi and T. Yanagida, Upper bound of the lightest Higgs boson mass in the minimal supersymmetric standard model, Prog. Theor. Phys. 85 (1991) 1 [INSPIRE].

[21] Y. Okada, M. Yamaguchi and T. Yanagida, Renormalization group analysis on the Higgs mass in the softly broken supersymmetric standard model, Phys. Lett. B 262 (1991) 54 [INSPIRE].

[22] J.R. Ellis, G. Ridolfi and F. Zwirner, Radiative corrections to the masses of supersymmetric Higgs bosons, Phys. Lett. B 257 (1991) 83 [INSPIRE].

[23] H.E. Haber and R. Hempfling, Can the mass of the lightest Higgs boson of the minimal supersymmetric model be larger than m(Z)?, Phys. Rev. Lett. 66 (1991) 1815 [INSPIRE].

[24] J.R. Ellis, G. Ridolfi and F. Zwirner, On radiative corrections to supersymmetric Higgs boson masses and their implications for LEP searches, Phys. Lett. B 262 (1991) 477 [InSPIRE].

[25] J. Hisano, T. Kuwahara and N. Nagata, Grand Unification in High-scale Supersymmetry, Phys. Lett. B 723 (2013) 324 [arXiv: 1304.0343] [InSPIRE].

[26] J. Hisano, D. Kobayashi, T. Kuwahara and N. Nagata, Decoupling Can Revive Minimal Supersymmetric SU(5), JHEP 07 (2013) 038 [arXiv:1304.3651] [INSPIRE].

[27] N. Nagata and S. Shirai, Sfermion Flavor and Proton Decay in High-Scale Supersymmetry, JHEP 03 (2014) 049 [arXiv: 1312.7854] [INSPIRE].

[28] J.L. Evans, N. Nagata and K.A. Olive, SU(5) Grand Unification in Pure Gravity Mediation, Phys. Rev. D 91 (2015) 055027 [arXiv: 1502.00034] [InSPIRE].

[29] Y. Nomura and S. Shirai, Supersymmetry from Typicality: TeV-Scale Gauginos and PeV-Scale Squarks and Sleptons, Phys. Rev. Lett. 113 (2014) 111801 [arXiv:1407.3785] [INSPIRE]. 
[30] L. Randall and R. Sundrum, Out of this world supersymmetry breaking, Nucl. Phys. B 557 (1999) 79 [hep-th/9810155] [INSPIRE].

[31] G.F. Giudice, M.A. Luty, H. Murayama and R. Rattazzi, Gaugino mass without singlets, JHEP 12 (1998) 027 [hep-ph/9810442] [INSPIRE].

[32] D.M. Pierce, J.A. Bagger, K.T. Matchev and R.-j. Zhang, Precision corrections in the minimal supersymmetric standard model, Nucl. Phys. B 491 (1997) 3 [hep-ph/9606211] [INSPIRE].

[33] A. Pomarol and R. Rattazzi, Sparticle masses from the superconformal anomaly, JHEP 05 (1999) 013 [hep-ph/9903448] [INSPIRE].

[34] A.E. Nelson and N.J. Weiner, Extended anomaly mediation and new physics at 10-TeV, hep-ph/0210288 [INSPIRE].

[35] K. Hsieh and M.A. Luty, Mixed gauge and anomaly mediation from new physics at 10-TeV, JHEP 06 (2007) 062 [hep-ph/0604256] [INSPIRE].

[36] A. Gupta, D.E. Kaplan and T. Zorawski, Gaugomaly Mediation Revisited, JHEP 11 (2013) 149 [arXiv: 1212.6969] [INSPIRE].

[37] K. Nakayama and T.T. Yanagida, Anomaly mediation deformed by axion, Phys. Lett. B 722 (2013) 107 [arXiv: 1302.3332] [INSPIRE].

[38] K. Harigaya, M. Ibe and T.T. Yanagida, A Closer Look at Gaugino Masses in Pure Gravity Mediation Model/Minimal Split SUSY Model, JHEP 12 (2013) 016 [arXiv:1310.0643] [INSPIRE].

[39] J.L. Evans and K.A. Olive, Universality in Pure Gravity Mediation with Vector Multiplets, Phys. Rev. D 90 (2014) 115020 [arXiv:1408.5102] [INSPIRE].

[40] J. Hisano, S. Matsumoto, M. Nagai, O. Saito and M. Senami, Non-perturbative effect on thermal relic abundance of dark matter, Phys. Lett. B 646 (2007) 34 [hep-ph/0610249] [INSPIRE].

[41] J. Hisano, S. Matsumoto and M.M. Nojiri, Explosive dark matter annihilation, Phys. Rev. Lett. 92 (2004) 031303 [hep-ph/0307216] [INSPIRE].

[42] J. Hisano, S. Matsumoto, M.M. Nojiri and O. Saito, Non-perturbative effect on dark matter annihilation and gamma ray signature from galactic center, Phys. Rev. D 71 (2005) 063528 [hep-ph/0412403] [INSPIRE].

[43] T. Cohen, M. Lisanti, A. Pierce and T.R. Slatyer, Wino Dark Matter Under Siege, JCAP 10 (2013) 061 [arXiv:1307.4082] [INSPIRE].

[44] J. Fan and M. Reece, In Wino Veritas? Indirect Searches Shed Light on Neutralino Dark Matter, JHEP 10 (2013) 124 [arXiv:1307.4400] [INSPIRE].

[45] M. Ibe, S. Matsumoto, S. Shirai and T.T. Yanagida, Wino Dark Matter in light of the AMS-02 2015 Data, Phys. Rev. D 91 (2015) 111701 [arXiv:1504.05554] [INSPIRE].

[46] K. Hamaguchi, T. Moroi and K. Nakayama, AMS-02 Antiprotons from Annihilating or Decaying Dark Matter, Phys. Lett. B 747 (2015) 523 [arXiv:1504.05937] [INSPIRE].

[47] J. Hisano, K. Ishiwata and N. Nagata, A complete calculation for direct detection of Wino dark matter, Phys. Lett. B 690 (2010) 311 [arXiv: 1004.4090] [INSPIRE].

[48] J. Hisano, K. Ishiwata and N. Nagata, Gluon contribution to the dark matter direct detection, Phys. Rev. D 82 (2010) 115007 [arXiv:1007.2601] [INSPIRE]. 
[49] J. Hisano, K. Ishiwata, N. Nagata and T. Takesako, Direct Detection of Electroweak-Interacting Dark Matter, JHEP 07 (2011) 005 [arXiv:1104.0228] [INSPIRE].

[50] J. Hisano, K. Ishiwata and N. Nagata, Direct Search of Dark Matter in High-Scale Supersymmetry, Phys. Rev. D 87 (2013) 035020 [arXiv:1210.5985] [InSPIRE].

[51] J. Hisano, K. Ishiwata and N. Nagata, QCD Effects on Direct Detection of Wino Dark Matter, JHEP 06 (2015) 097 [arXiv: 1504.00915] [INSPIRE].

[52] M. Ibe, T. Moroi and T.T. Yanagida, Possible Signals of Wino LSP at the Large Hadron Collider, Phys. Lett. B 644 (2007) 355 [hep-ph/0610277] [INSPIRE].

[53] S. Asai, T. Moroi and T.T. Yanagida, Test of Anomaly Mediation at the LHC, Phys. Lett. B 664 (2008) 185 [arXiv:0802.3725] [INSPIRE].

[54] S. Asai, Y. Azuma, O. Jinnouchi, T. Moroi, S. Shirai and T.T. Yanagida, Mass Measurement of the Decaying Bino at the LHC, Phys. Lett. B 672 (2009) 339 [arXiv:0807.4987] [INSPIRE].

[55] K. Griest and D. Seckel, Three exceptions in the calculation of relic abundances, Phys. Rev. D 43 (1991) 3191 [INSPIRE].

[56] N. Nagata, H. Otono and S. Shirai, Probing bino-gluino coannihilation at the LHC, Phys. Lett. B 748 (2015) 24 [arXiv: 1504.00504] [INSPIRE].

[57] H. Baer, T. Krupovnickas, A. Mustafayev, E.-K. Park, S. Profumo and X. Tata, Exploring the BWCA (bino-wino co-annihilation) scenario for neutralino dark matter, JHEP 12 (2005) 011 [hep-ph/0511034] [INSPIRE].

[58] N. Arkani-Hamed, A. Delgado and G.F. Giudice, The Well-tempered neutralino, Nucl. Phys. B 741 (2006) 108 [hep-ph/0601041] [INSPIRE].

[59] M. Ibe, A. Kamada and S. Matsumoto, Mixed (cold+warm) dark matter in the bino-wino coannihilation scenario, Phys. Rev. D 89 (2014) 123506 [arXiv:1311.2162] [INSPIRE].

[60] K. Harigaya, K. Kaneta and S. Matsumoto, Gaugino coannihilations, Phys. Rev. D 89 (2014) 115021 [arXiv:1403.0715] [INSPIRE].

[61] H.E. Haber and D. Wyler, Radiative neutralino decay, Nucl. Phys. B 323 (1989) 267 [INSPIRE].

[62] S. Ambrosanio and B. Mele, Supersymmetric scenarios with dominant radiative neutralino decay, Phys. Rev. D 55 (1997) 1399 [Erratum ibid. D 56 (1997) 3157] [hep-ph/9609212] [INSPIRE].

[63] M.A. Diaz, B. Panes and P. Urrejola, Radiative Neutralino Decay in Split Supersymmetry, Eur. Phys. J. C 67 (2010) 181 [arXiv:0910.1554] [INSPIRE].

[64] C. Han, L. Wu, J.M. Yang, M. Zhang and Y. Zhang, New approach for detecting a compressed bino/wino at the LHC, Phys. Rev. D 91 (2015) 055030 [arXiv:1409.4533] [INSPIRE].

[65] T. Takagi, On an Algebraic Problem Reluted to an Analytic Theorem of Carathéodory and Fejér and on an Allied Theorem of Landau, Jpn. J. Math. 1 (1924) 83.

[66] S.Y. Choi, H.E. Haber, J. Kalinowski and P.M. Zerwas, The Neutralino sector in the U(1)-extended supersymmetric standard model, Nucl. Phys. B 778 (2007) 85 [hep-ph/0612218] [INSPIRE]. 
[67] Y. Yamada, Electroweak two-loop contribution to the mass splitting within a new heavy $\mathrm{SU}(2)_{L}$ fermion multiplet, Phys. Lett. B 682 (2010) 435 [arXiv:0906.5207] [INSPIRE].

[68] M. Ibe, S. Matsumoto and R. Sato, Mass Splitting between Charged and Neutral Winos at Two-Loop Level, Phys. Lett. B 721 (2013) 252 [arXiv:1212.5989] [INSPIRE].

[69] ATLAS collaboration, Search for direct production of charginos, neutralinos and sleptons in final states with two leptons and missing transverse momentum in pp collisions at $\sqrt{s}=8$ TeV with the ATLAS detector, JHEP 05 (2014) 071 [arXiv: 1403.5294] [INSPIRE].

[70] CMS collaboration, Searches for electroweak production of charginos, neutralinos and sleptons decaying to leptons and $W, Z$ and Higgs bosons in pp collisions at 8 TeV, Eur. Phys. J. C 74 (2014) 3036 [arXiv:1405.7570] [InSPIRE].

[71] J.L. Evans, M. Ibe, K.A. Olive and T.T. Yanagida, Light Higgsinos in Pure Gravity Mediation, Phys. Rev. D 91 (2015) 055008 [arXiv:1412.3403] [InSPIRE].

[72] N. Nagata and S. Shirai, Higgsino Dark Matter in High-Scale Supersymmetry, JHEP 01 (2015) 029 [arXiv: 1410.4549] [INSPIRE].

[73] J. Ellis, F. Luo and K.A. Olive, Gluino Coannihilation Revisited, JHEP 09 (2015) 127 [arXiv: 1503.07142] [INSPIRE].

[74] ATLAS collaboration, Search for massive, long-lived particles using multitrack displaced vertices or displaced lepton pairs in pp collisions at $\sqrt{s}=8 \mathrm{TeV}$ with the ATLAS detector, arXiv: 1504.05162 [INSPIRE].

[75] ATLAS collaboration, Search for long-lived, heavy particles in final states with a muon and a multi-track displaced vertex in proton-proton collisions at $\sqrt{s}=8 \mathrm{TeV}$ with the ATLAS detector, ATLAS-CONF-2013-092 (2013).

[76] ATLAS collaboration, Search for long-lived, heavy particles in final states with a muon and multi-track displaced vertex in proton-proton collisions at $\sqrt{s}=7$ TeV with the ATLAS detector, Phys. Lett. B 719 (2013) 280 [arXiv:1210.7451] [inSPIRE].

[77] ATLAS collaboration, Search for displaced vertices arising from decays of new heavy particles in $7 \mathrm{TeV}$ pp collisions at ATLAS, Phys. Lett. B 707 (2013) 478 [arXiv:1109.2242] [INSPIRE].

[78] CMS collaboration, Search for Long-Lived Neutral Particles Decaying to Quark-Antiquark Pairs in Proton-Proton Collisions at $\sqrt{s}=8$ TeV, Phys. Rev. D 91 (2015) 012007 [arXiv:1411.6530] [INSPIRE].

[79] ATLAS collaboration, Search for nonpointing and delayed photons in the diphoton and missing transverse momentum final state in $8 \mathrm{TeV}$ pp collisions at the LHC using the ATLAS detector, Phys. Rev. D 90 (2014) 112005 [arXiv:1409.5542] [InSPIRE].

[80] CMS collaboration, Search for long-lived particles that decay into final states containing two electrons or two muons in proton-proton collisions at $\sqrt{s}=8 \mathrm{TeV}$, Phys. Rev. D 91 (2015) 052012 [arXiv: 1411.6977] [INSPIRE].

[81] CMS collaboration, Search for Displaced Supersymmetry in events with an electron and a muon with large impact parameters, Phys. Rev. Lett. 114 (2015) 061801 [arXiv:1409.4789] [INSPIRE].

[82] ATLAS collaboration, Search for pair-produced long-lived neutral particles decaying in the ATLAS hadronic calorimeter in pp collisions at $\sqrt{s}=8 \mathrm{TeV}$, Phys. Lett. B 743 (2015) 15 [arXiv: 1501.04020] [INSPIRE]. 
[83] ATLAS collaboration, Search for long-lived, weakly interacting particles that decay to displaced hadronic jets in proton-proton collisions at $\sqrt{s}=8 \mathrm{TeV}$ with the ATLAS detector, Phys. Rev. D 92 (2015) 012010 [arXiv: 1504.03634] [INSPIRE].

[84] J. Alwall, M. Herquet, F. Maltoni, O. Mattelaer and T. Stelzer, MadGraph 5: Going Beyond, JHEP 06 (2011) 128 [arXiv:1106.0522] [INSPIRE].

[85] T. Sjöstrand, S. Mrenna and P.Z. Skands, PYTHIA 6.4 Physics and Manual, JHEP 05 (2006) 026 [hep-ph/0603175] [INSPIRE].

[86] DELPHES 3 collaboration, J. de Favereau et al., DELPHES 3, A modular framework for fast simulation of a generic collider experiment, JHEP 02 (2014) 057 [arXiv:1307.6346] [INSPIRE].

[87] W. Beenakker, R. Hopker and M. Spira, PROSPINO: A program for the production of supersymmetric particles in next-to-leading order QCD, hep-ph/9611232 [INSPIRE].

[88] J. Hisano, D. Kobayashi, N. Mori and E. Senaha, Effective Interaction of Electroweak-Interacting Dark Matter with Higgs Boson and Its Phenomenology, Phys. Lett. B 742 (2015) 80 [arXiv: 1410.3569] [INSPIRE].

[89] N. Nagata and S. Shirai, Electroweakly-Interacting Dirac Dark Matter, Phys. Rev. D 91 (2015) 055035 [arXiv: 1411.0752] [INSPIRE].

[90] J.F. Gunion and S. Mrenna, Probing models with near degeneracy of the chargino and LSP at a linear $e^{+} e^{-}$collider, Phys. Rev. D 64 (2001) 075002 [hep-ph/0103167] [INSPIRE].

[91] K. Harigaya, K. Ichikawa, A. Kundu, S. Matsumoto and S. Shirai, Indirect Probe of Electroweak-Interacting Particles at Future Lepton Colliders, JHEP 09 (2015) 105 [arXiv: 1504.03402] [INSPIRE].

[92] K. Rolbiecki and K. Sakurai, Long-lived bino and wino in supersymmetry with heavy scalars and higgsinos, arXiv:1506.08799 [INSPIRE]. 